Maria Roca Lizarazu

\title{
Liaisons Dangereuses: Nachbarn, (Mit-)Täter und implicated subjects in Katja Petrowskajas Vielleicht Esther
}

\section{Einleitung}

Katja Petrowskajas autobiografisch inspiriertes Debüt Vielleicht Esther (2014) ${ }^{1}$ erzählt eine Familiengeschichte, die sich über unterschiedliche Epochen, diverse nationale und kulturelle Grenzen sowie verschiedene sprachliche und mediale Räume erstreckt: Die in der Ukraine geborene und zu Beginn des Textes in Berlin lebende Ich-Erzählerin begibt sich auf eine Odyssee, die sie zuerst ins Polen des neunzehnten Jahrhunderts führt, wo sie die Geschichte ihres Großvaters mütterlicherseits zu ergründen sucht, der seinerzeit mehrere Taubstummenschulen für jüdische Waisen gegründet hat. Je tiefer sich die Erzählerin jedoch ins Dickicht ihrer Familiengeschichte begibt, desto deutlicher tritt zu Tage, dass ihre persönliche Familienbiografie sich nicht vom Panorama (ost-)europäischer Geschichte im neunzehnten und zwanzigsten Jahrundert ablösen lässt und dass sie, als Jüdin, nicht umhinkann, sich mit den Nachwirkungen des Holocausts auseinanderzusetzen. Ihre Recherche- und Suchbewegungen, die an mittlerweile weit verbreitete Motive und Topoi zahlreicher sogenannter „Familien-“ oder „Generationenromane" (Costagli und Galli 2010; Eichenberg 2009; Eigler 2005; Fuchs 2008; Herrmann 2010; Horstkotte 2009; Reidy 2013; Weigel 2006) anschließen, werden wiederholt unterbrochen und/oder laufen ins Leere; dies ist zum einen den (un-)willentlichen Lücken in ihrem Familiengedächtnis und zum anderen der problematischen Gedächtnispolitik in der (post-)sowjetischen Ära geschuldet. Im Laufe der Erzählung wird deutlich, dass die zahlreichen Streichungen im individuellen und kollektiven Gedächtnis unmöglich zu kompensieren sind - statt die Leerstellen im persönlichen und im offiziellen Narrativ phantasmatisch aufzufüllen, entscheidet sich die Erzählerin deshalb dafür, im Modus des metafiktionalen Schreibens die Spannungen zwischen Erinnern und Vergessen, Persönlichem und Privatem, Historiografie und (Auto-)Biografie, Fakt und Fiktion zu reflektieren. Die Frucht dieser Überlegungen ist eine Sammlung von Geschichten, so der Untertitel des Textes Vielleicht Esther, der explizit die Gattungszuschreibung „Roman“ ablehnt. Dies hat möglicherweise mit der diese

1 Im Folgenden abgekürzt zitiert mit der Sigle VE, Seitenangabe. 
Gattung prägende „Gesinnung zur Totalität“ zu tun, um Georg Lukács zu zitieren (1916, 245), verstanden als tendenzielles Streben nach Geschlossenheit, das einhergeht mit teleologischen Erzählmustern. ${ }^{2}$ Demgegenüber betont der Untertitel Geschichten die Offenheit, Pluralität und Kontingenz historischer Ereignisse und Erfahrungen.

Petrowskajas Text begeisterte nicht nur die Kritiker, die den titelgebenden Textauschnitt „Vielleicht Esther“ mit dem prestigeträchtigen Ingeborg-Bachmann-Preis auszeichneten, sondern sorgte auch in der germanistischen Forschung für Aufsehen. Gemessen an der kurzen Publikationsgeschichte des im Jahre 2014 erschienenen Textes, hat Vielleicht Esther bereits ein beachtliches Maß an akademischer Beachtung gefunden (Bühler-Dietrich 2019; Eckart 2015; Ortner 2017; Osborne 2016; Perrone Capano 2018; Roca Lizarazu 2018; Tzschentke 2015; Vestli 2016; Weiss-Sussex 2017). Die vorhandenen Forschungsbeiträge setzen sich mit Gedächtniskonstruktionen und Erinnerungsdiskursen (Osborne 2016; Ortner 2017; Perrone Capano 2018; Roca Lizarazu 2018; Tzschentke 2015), Fragen von Identität und Migration (Eckart 2015; Perrone Capano 2018; Vestli 2016) oder auch den Besonderheiten von Petrowskajas Sprachgebrauch (Bühler-Dietrich 2019; Eckart 2015; Weiss-Sussex 2017) auseinander.

Obwohl viele der oben genannten Beiträge Fragen nach Opfertum und, in geringerem Maße, nach Täterschaft streifen, ${ }^{3}$ steht eine explizite Auseinandersetzung mit Opfer- und Täterdiskursen in Vielleicht Esther bislang noch aus. Der vorliegende Beitrag will sich diesem zentralen Thema über eine „Figur des Dritten“ (Eßlinger et al. 2010) nähern, indem Nachbar^innen und Nachbarschaftsverhältnisse in Petrowskajas Text näher betrachtet und untersucht werden sollen. Im Kontext von Opfer- und Täterkonstellationen des Zweiten Weltkriegs und des Holocausts, wie sie für Vielleicht Esther prägend sind, ist die Figur des Nachbarn besonders interessant, da er*sie - ganz im Sinne einer „Figur des Dritten“ - „Probleme der Grenzziehung, des Übergangs und der Vermischung zwischen opponierenden Bedeutungsfeldern“ (Koschorke 2010, 11) aufwirft. Der Nachbar in Vielleicht Esther fungiert als höchst ambivalente Figur, die zwischen den Polen

2 Es sollte an dieser Stelle angemerkt werden, dass Lukács' Romantheorie von der Spannung zwischen dem tendenziellen Totalitätsanspruch des Romans und der Unmöglichkeit von Totalitätserfahrungen in der Moderne durchzogen ist. Auf gewisse Weise bildet für Lukács die Aushandlung dieser Spannung den Kern der Gattungsform Roman: „Der Roman ist die Epopöe eines Zeitalters, für das die extensive Totalität des Lebens nicht mehr sinnfällig gegeben ist, für das die Lebensimmanenz des Sinnes zum Problem geworden ist, und das dennoch die Gesinnung zur Totalität hat“ (1916, 245).

3 Eine Ausnahme stellt Dora Osbornes Beitrag dar, der sich explizit mit dem Zusammenhang von Archiv, Zeugschaft, Täterschaft und Komplizenschaft auseinandersetzt (2016). 
von Freund und Feind, des Eigenen und Fremden sowie von Innen und Außen changiert bzw. deren Grenzen verwischt.

Im Folgenden geht dieser Beitrag daher von der These aus, dass Nachbarschaft in Vielleicht Esther untrennbar mit Fragen der Komplizen- und Mitläuferschaft und der implication verbunden ist, die nach Michael Rothberg als „modes of historical relation that do not necessarily fall under the more direct forms of participation associated with traumatic events, such as victimisation and perpetration“ (2013, 40), verstanden werden können. Rothberg führte den Begriff der implication in aktuelle Debatten um Holocaust-Erinnerung ein, um auf das Problem zunehmender historischer Distanz einzugehen (2013, 2014a, 2014b). Nach Meinung Rothbergs muss der Fokus auf direkte Teilnahme an den Verbrechen und aktive Täterschaft, der für gegenwärtige und kommende Generationen immer weniger relevant ist, durch einen strukturellen Ansatz ersetzt werden, der „the conditions of possibility of violence as well as its lingering impact“ (2014b) zum Ziel hat und also jene (möglicherweise fortbestehenden) Grundbedingungen reflektiert, die die Gewalttaten des Holocausts (und anderer Genozide) überhaupt erst ermöglicht haben. ${ }^{4}$ Implication bietet sich daher als Konzept an, um vermittelte Formen der Teilnahme, Täterschaft oder auch der Mittäter- und Nutznießerschaft, die sich jenseits der Binäropposition von Opfer und Täterinnen abspielen, zu untersuchen und zugleich die Langzeiteffekte großflächiger Gewalterfahrungen in den Fokus zu rücken. Insofern lässt sich an Figuren der Nachbarschaft und der implication auch überprüfen, inwieweit Vielleicht Esther den Anspruch der Autorin Katja Petrowskaja umsetzt, die von ihr als unproduktiv empfundenen Kategorien „Opfer“ und „Täter“ zu verabschieden: „Und so konnte ich davon erzählen, dass die Geschichte von Opfer und Täter für mich passé ist. Wenn man die Rollenfestlegung immer weiter trägt, dann bleibt man unweigerlich darin stecken, ohne etwas zu verstehen“ (Heimann 2013). ${ }^{5}$ Gleichzeitig wirft Rothbergs Konzept die wichtige Frage auf, wie sich ein solcher struktureller Ansatz erzählen und erinnern lässt und hier wiederum bieten die Literatur, und künstlerische Diskurse im Allgemeinen, mögliche Ansätze.

\footnotetext{
4 Max Silverman denkt in eine ähnliche Richtung, er verwendet allerdings den Begriff der complicity, i.e. der Komplizenschaft (2010).

5 Darüber hinaus ist die Figur des Nachbarn - und insbesondere der Aufruf, diesen zu lieben, wie sich selbst - nicht nur für das judäo-christliche Wertesystem zentral, wie Slavoj Žižek, Eric L. Santner und Kenneth Reinhard aufgezeigt haben (2005), sondern auch von enormer Wichtigkeit für den deutsch-jüdischen Diskurs seit dem Mittelalter. Bisher gibt es keine systematische Aufarbeitung dieser Konstellation in der deutsch-jüdischen Debatte.
} 
Im Folgenden sollen deshalb Figuren des Nachbarn und Figurationen von Nachbarschaft im Text in Bezug auf folgende zentrale Problemstellungen untersucht werden:

Täterschaft und Komplizenschaft: Es soll zum einen gezeigt werden, wie die Figur des Nachbarn die Trennlinie von „wir“ und „anderen“ (VE, 105) destabilisiert und damit sowohl den Opfer- als auch den Täterstatus ,verunklart`, nicht zuletzt, indem das im osteuropäischen Raum nach wie vor tabuisierte Thema der Kollaboration und Komplizenschaft aufgerufen wird.

Erinnerung und Zeugenschaft: Indem Vielleicht Esther die Binäropposition ,Täter`innen‘/,Opfer‘ mit einem verflochtenen oder „implicated“ (Rothberg 2013) Ansatz kontrastiert, ergeben sich neuartige Probleme für das Erinnern der Verbrechen des Zweiten Weltkriegs und des Holocausts. Wie lässt sich die nach wie vor bestehende kulturelle, mnemonische und räumliche Verflechtung zwischen jüdischem und nicht-jüdischem Leben und Sterben erzählen und können Kompliz ${ }^{\star}$ innen, Mitläufer^innen, Nachbar^innen und Passant`innen Zeugnis ablegen?

Kontiguität und Communitas: Schlussendlich will dieser Beitrag auch auf die möglichen positiven Konnotationen von Nachbarschaft im Text eingehen; neben Nachbar`innen, die helfen und retten, verhandelt Vielleicht Esther über das Thema Nachbarschaft - verstanden im Sinne von Kontiguität, also des räumlichen Nebeneinanders - Formen der (Erinnerungs-)Gemeinschaft, die auf Kontingenz, Flüchtigkeit und den für den Text wichtigen Metaphern der Verwobenheit oder Überkreuzung beruhen und damit die essentialisierende Logik des familiär und ethnisch zentrierten Gedächtnisses im Text auf produktive Weise in Frage stellen.

\section{Opfer, Täter*innen und alles, was dazwischen liegt}

Gedanken zum Thema Nachbarschaft durchziehen Petrowskajas gesamten Text, werden jedoch explizit in den Kapiteln zum Polenbesuch der Erzählerin und zu ihrer Begegnung mit der ukrainischen Schlucht Babij Jar verhandelt. Beide Länder, Polen und die Ukraine, sind geprägt durch eine lange Geschichte des Antisemitismus und, damit verbunden, nach wie vor anhaltende gedächtnispolitische Debatten über das Ausmaß der Kollaboration während des Zweiten Weltkriegs und des Holocausts; das spannungsreiche Zusammenleben von Jüdinnen und Nicht-Jüd*innen ist also zentral für beide Nationen, ebenso wie anhaltende Konflikte um eine angemessene Erinnerung an Opfer und (Mit-)Täterinnen des Zweiten Weltkriegs und des Holocaust. Für die Erzählerin jedoch scheint sich 
das Thema Nachbarschaft auch aus der geografischen Lage beider Länder zu ergeben. Polen, an die Ukraine grenzend, wurde von der Erzählerin schon immer als begehrenswerte, jedoch zugleich unnahbare Nachbarin wahrgenommen: „Als ich in Kiew aufwuchs, war Polen, unser nächster Nachbar, auf Russisch Polscha, unsere Nachbarin, ein unerreichbares schönes Ausland“ (VE, 91). Die Polenreise der Erzählerin trägt unter anderem dazu bei, dieses romantisierende Polen-Bild, das auf ihren Vater zurückgeht ( $V E$, 92), nach und nach zu demontieren und die mit dem Thema Nachbarschaft verbundenen Ambivalenzen hervortreten $\mathrm{zu}$ lassen: Polen ist auch das Land, in dem Oświęcim (i.e. die Stadt, in der sich das Konzentrationslager Auschwitz befindet) und das Warschauer Ghetto liegen, ${ }^{6}$ und das Verhältnis zwischen Pol*innen und Jüdinnen vor, während des und nach dem Holocaust ist nach wie vor Gegenstand hitziger historiografischer und gedenkpolitischer Diskussionen, wie sich beispielsweise an der Kontroverse um die Studie Neighbours des Historikers Jan T. Gross (2001) und erst kürzlich wieder in den Debatten um die Einführung des sogenannten „Holocaust-Gesetzes“ zeigte. $^{7}$

Die Erzählerin begibt sich also auf gedächtnispolitisch vermintes Terrain, welches sie jedoch als eine auf Deutsch schreibende und in Deutschland lebende ukrainische Jüdin, die sich sowohl als Vertreterin des Opferkollektivs (d.h. des jüdischen Volkes) als auch der Tätergruppierungen (d.h. der ehemaligen Sowjetmacht) begreift, möglicherweise anders durchmessen kann. Wenngleich die Themen Komplizenschaft und Kollaboration für ihre Begegnung mit Polen und Babij Jar wichtig sind, dient ihr das Thema Nachbarschaft doch zuerst einmal dazu, historisch abstrakte Kategorien und Binäroppositionen, wie beispielsweise ,Opfer‘ vs. ,Täter`innen‘, ,Jüdinnen` vs. ,Nicht-Jüd^innen` und ,wir‘ vs. ,andere‘,

6 Petrowskajas Erzählerin verwendet bewusst die im deutschen Kontext eher weniger gebräuchliche polnische Bezeichnung für das Vernichtungslager Auschwitz (siehe VE, 57-58). Wie Godela Weiss-Sussex richtigerweise angemerkt hat, hat dies zum einen mit einem Widerstand gegen die Formelhaftigkeit der Chiffre Auschwitz zu tun; die Verwendung des polnischen Namens erzeugt somit eine Art Verfremdungseffekt (2017). Im Kontext des vorliegenden Beitrags könnte die Verwendung des polnischen Namens jedoch auch auf die Thematik polnischer (Mit-)Täterschaft oder zumindest Mitverantwortung verweisen, die durch den Gebrauch des deutschen Namens möglicherweise ausgeblendet wird. Um genau jene Fragen der Bezeichnung und (Mit-)Verantwortung ging es interessanterweise auch während der Debatten um das umstrittene „HolocaustGesetz“, das unter anderem die Verwendung des Begriffs „polnische Konzentrationslager“ unter Strafe stellen sollte, siehe hierzu Harper 2016.

7 Anfang 2018 führte die derzeitige polnische Regierung ein Gesetz ein, das es verbietet, dem polnischen Staat Mitverantwortung an den Verbrechen der Nationalsozialisten zuzuschreiben. Das Gesetz löste zahlreiche, auch internationale, Kontroversen aus und wurde daraufhin im Sommer 2018 abgemildert, siehe Dudek 2018; Sierdaka 2018. 
zu destabilisieren. In den Augen der Erzählerin sind diese verallgemeinernden Zuschreibungen problematisch, da sie es nicht nur den damals Beteiligten, sondern auch den heute in diesen Gesellschaften Lebenden leichter machen, das Geschehene von sich abzuspalten. Zugleich haben diese Kategorien einen naturalisierenden Effekt, da sie die Trennung zwischen ,wir‘ und ,anderen“ als schon immer gegeben erscheinen lassen und gleichzeitig eine Assoziationskette aus ,Jüdinnen“ - ,die anderen“ - ,Opfer‘ perpetuieren. Eben jene essentialisierenden Logiken hinterfragt Vielleicht Esther jedoch wiederholt, da sie verschleiern helfen, dass sich die Gewaltereignisse des zwanzigsten Jahrhunderts nicht schicksalhaft, sondern durch die (Nicht-)Taten und Entscheidungen vieler Einzelner entfaltet haben: „[...] ich sage auch oft natürlich oder sogar naturgemäß, als ob dieses Verschwinden oder dieses Nichts natürlich oder auch selbstverständlich sei“ (VE, 10). Das bereits im Titel angedeutete konjunktivische Erzählprogramm verweist darauf, dass ein anderer Ausgang der Geschichte zwar nicht gegeben ist, aber möglich gewesen wäre. Schließlich begünstigt das Denken in den oben genannten Mustern einen abstrahierenden Blick, der das konkrete (Zusammen-) Leben und Sterben aller Beteiligten ausblendet und zugleich den Blick auf das Weiterleben versperrt, denn wenn ,Jüd`innen“ kategorisch mit ,Opfern` gleichgesetzt werden, werden sie - zumindest implizit - immer auch als tote und nicht als lebendige Gemeinschaft gedacht.

Wenn man sich jedoch bewusst macht, dass Opfer und Täter*innen in vielen Fällen Nachbar^innen waren, lassen sich diese sauberen Trennungen und die bequeme Distanz zum Geschehenen nicht aufrechterhalten:

Ja, man nennt diese Opfer für gewöhnlich Juden, aber viele meinen damit nur die anderen. Das ist irreführend, denn die, die da sterben mussten, waren nicht die anderen, sondern die Schulfreunde, die Kinder aus dem Hinterhof, die Nachbarn, die Omas und die Onkel [...]. (VE, 185)

Durch den Verweis auf Nachbarschaftsverhältnisse durchbricht Petrowskajas Text den Abwehrmechanismus, der ,Opfer‘ mit ,Jüd^innen“ und den ,anderen` gleichsetzt. Die Jüd^innen waren eben „nicht die anderen“ $(V E, 185)$, sondern verflochten mit und Teil der Gemeinschaft; die Trennung zwischen ,wir` und ,anderen“ wird dadurch nicht nur als Ausgangspunkt der gewaltsamen Vernichtung, sondern auch als Produkt einer bestimmten Gedächtnispolitik im osteuropäischen Raum sichtbar, die die jüdischen Opfer nach wie vor als „die Toten der ewigen anderen“ (VE, 190) oder als die „Waisen unserer gescheiterten Erinnerung“ (VE, 191) segregiert. In dem Maße, in dem die ,Jüd`innen` vom Status der Andersheit abgekoppelt werden, wird es auch im Falle der ,Täter`innen‘ zunehmend schwerer, diese im kollektiven Gedächtnis als ,die anderen' (i.e. als die Deutschen) vom Rest der Bevölkerung abzuspalten. Wenn Opfer wie Täter^innen Nachbar^innen waren, impliziert dies 
auch, dass die Zivilbevölkerung zumindest teilweise in die Verbrechen verstrickt war. Durch räumliche Nähe wird also ein Moment der Intimität und der Verflechtung eingeführt, das eine Reihe unangenehmer Fragen aufwirft:

Zum einen verdeutlicht Vielleicht Esther, dass diese Intimität durchaus täuschend sein kann, mitunter gar tödlich endet. Während ihres Besuchs in Warschau liest die Erzählerin ein Theaterstück, das vermutlich auf der bereits erwähnten skandalträchtigen Studie Neighbours (2001) von Jan T. Gross basiert. Gross widmet sich dem Jedwabne-Pogrom, bei dem im Jahr 1941 mehrere hundert - nach Angaben von Gross sogar 1600 - Jüd*innen von ihren polnischen Mitbürger^innen auf grausame Weise hingerichtet wurden. Laut Gross ging das Massaker vor allem von polnischer und nicht von der Seite der deutschen Besatzungsmacht aus. Die Studie löste vor allem in Polen heftige Debatten aus, die sich nicht nur um die lange Geschichte antisemitischer Ausschreitungen im Land, sondern auch um das tabuisierte Thema polnischer Mittäterschaft und Komplizenschaft in Bezug auf diverse NS-Verbrechen drehten (Polonsky und Michlic 2004). Die Auseinandersetzung mit dem Stück und dem ihm zugrundeliegenden Pogrom erweckt in der Erzählerin ein Bewusstsein für die Ambivalenzen der Nachbarschaft, die nicht nur das Verhältnis zur schönen Nachbarin Polscha, sondern auch zu ihrem Berliner Nachbarn, dem sie in Warschau zufällig über den Weg läuft, zu trüben scheinen. An sich wäre dieses Treffen

[...] ein schöner Zufall gewesen [...], hätte ich nicht gerade ein Theaterstück gelesen, das sich auf ein Buch mit dem Titel Nachbarn bezieht und in dem es um Klassenkameraden geht, Polen und Juden, die zusammen aufwuchsen, zusammen lebten, einander mochten und sich dann gegeneinander wendeten und einander töteten, wer wen, just guess, und gerade dachte ich an die Nachbarn in einer kleinen polnischen Stadt mit dem für mich unaussprechlichen Namen Jedwabne und warum man seine Nachbarn tötet, im Delirium, in der Finsternis, im Affekt oder auch gerne, da stand er plötzlich vor mir [...]. (VE, 109-110)

Diese Unberechenbarkeit des Nachbarschaftsverhältnisses wird auch im Kapitel zur titelgebenden Urgroßmutter der Erzählerin wieder aufgenommen, in dem die Nachbar*innen „Vielleicht Esther“ einerseits mit Essen versorgen (VE, 209) und versuchen, vom Gang zur Schlucht von Babij Jar abzuhalten $(V E, 211),{ }^{8}$ gleichzei-

8 Der Name „Vielleicht Esther“ für die Urgroßmutter der Erzählerin leitet sich zum einen daraus her, dass der Vater der Erzählerin sich nicht mehr genau an den Namen erinnern kann: „Ich glaube, sie hieß Esther, sagte mein Vater. Ja, vielleicht Esther“ (VE, 209). Zum anderen steht das „vielleicht“ im Namen der Urgroßmutter aber auch im erweiterten Sinne für das gesamte Erzählprogramm des Romans, das sich nicht nur um das Zusammenspiel zwischen (fehlenden) historischen Fakten und Fiktion dreht, sondern auch um die im Adverb „vielleicht“ angedeuteten Ungewissheiten, Ungereimtheiten und uneingelösten Möglichkeiten historischer Erfahrung. 
tig jedoch auch diejenigen sind, die ihr die Treppe herunter- und auf die Straße helfen und sie so - wissentlich oder nicht - in den Tod schicken (VE, 211).

Neben diesem unheimlichen Effekt, der das Vertraute suspekt erscheinen lässt, hat die Fokussierung auf die soziale Mikro-Ebene der Nachbarschaftsverhältnisse eine weitere Folge: Indem die abstrakten Kategorien von ,Opfer`,Täter`innen`, ,die anderen'/,wir' in konkrete Nachbarschaftsverhältnisse übersetzt werden, wird die Vernichtung paradoxerweise nicht greifbarer, sondern unvorstellbarer, denn die geläufige und vereinfachende Binäropposition Opfer vs. Täter`innen wird ersetzt durch ein Netzwerk der implication (Rothberg 2014a, 2014b), das Jüdinnen, Pol`innen, Deutsche, Opfer, Täter`innen, Mitläufer^innen, Beobachter^innen usw. einbindet und dessen Reichweite sich nur schwer vor- und darstellen lässt, wie Rothberg in Bezug auf den südafrikanischen Künstler William Kentridge und dessen Auseinandersetzung mit den Nachwirkungen des Apartheitsregimes gezeigt hat (Rothberg 2013). Dies hat nicht zuletzt damit zu tun, dass die Frage, wo eine (Un-)Tat und Täterschaft beginnt, aus Perspektive der implication neu zu stellen ist: Zählen die Nachbar^innen, die „Vielleicht Esther“ die Treppe herunterhelfen als (Mit-)Täter^innen? Wie viel Schuld und Verantwortung tragen all jene, die „hinter den Vorhängen“ (VE, 222) zuschauen und nichts tun, und wie lassen sich die „Bauern, Priester [...], Menschen, damals noch Kinder [...]“ $(V E, 272)$ beschreiben, die aus variierender Nähe und Distanz am Todesmarsch ungarischer Jüd *innen von Mauthausen nach Gunskirchen im April 1945 teilnahmen?

In den Verhandlungen von Nachbarschaft und implication im Text schwingt demnach auch die grundlegende Frage mit, ob es angesichts der Verbrechen des zwanzigsten Jahrhunderts eine ,unschuldige‘, im Sinne von unbeteiligte oder nicht kompromittierte, Position überhaupt geben kann. Das im Text wiederkehrende Paradiesapfel-/Sündenfall-Motiv oder auch die biblische Referenz auf den Brudermord von Kain scheinen die Möglichkeit der Schuldlosigkeit zu verneinen: „Wenn Kain Abel getötet hat und Abel keine Kinder hatte, wer sind dann wir?“ (VE, 251)

\section{Implication erinnern, erzählen, bezeugen}

Während es im vorherigen Abschnitt darum ging, wie das Thema Nachbarschaft Kategorien wie ,Opfer`/,Täterinnen`; ,Jüdinnen`/,Nicht-Jüdinnen`; ,wir`/,andere‘ in einer dichotomen Entgegensetzung destabilisiert und gängige Konzeptionen von Täterschaft, Schuld und Verantwortung hinterfragt, soll es im Folgenden um die Erinner- und Erzählbarkeit der „implicated“ Perspektive gehen, die nicht von Binaritäten, sondern von Nachbarschaftlichkeit und Verflochtenheit ausgeht. Diese Verstrickungen stellen eine Herausforderung für das Gedenken an die Verbrechen dar, da nicht nur unklar ist, wem Opfer und 
Täter^innen ,gehören‘, sondern auch, wie das Verhältnis von Kontinuität und Diskontinuität zu denken ist:

Im Jahr 1939, als der Krieg begann, lebte eine Million Menschen in Warschau, neununddreißig Prozent davon Juden [...], diese Neununddreißig veränderte für mich alles. Bei neununddreißig geht es nicht mehr um wir und die anderen, sondern um dich und deinen Nachbarn, dachte ich, um jeden zweiten oder dritten, um dich und mich [...]. Wie soll man der Hälfte der Stadt gedenken? Und wie kann man hier noch leben? Wenn man wie in Berlin für jeden Menschen einen Stolperstein der Erinnerung in den Bürgersteig einlassen würde, wären die Gassen und Straßen von Warschau mit goldenen Steinen gepflastert. (VE, 105)

Indem die Erzählerin fragt, wie man dieser „Hälfte der Stadt“ $(V E, 105)$ gedenken solle, wirft sie das Thema der Erinnerbarkeit auf, die in diesem Falle nicht nur durch Quantitäten gefährdet ist, d.h. die schiere Masse der zu erinnernden Opfer, die ihren Ausdruck in den hypothetischen, mit Goldsteinen gepflasterten Straßen Warschaus findet. Es geht auch um das Verhältnis von Kontinuität und Diskontinuität, das zentral für die Problematik der implication ist. Denn eine „implicated“ Perspektive auf den Holocaust erweitert nicht nur das Spektrum zwischen ,Opfer‘ und ,Täter`innen‘ um diverse Grauzonen, sondern geht auch von einer unumstößlichen Verflochtenheit von Vergangenheit, Gegenwart und Zukunft aus, die eben keinen endgültigen Schlussstrich zwischen heute und gestern zulässt. Gerade diese Verstrickungen und Kontinuitäten werden jedoch nach Meinung der Erzählerin im polnischen Kontext systematisch verneint, in dem die „Spuren dieses [des jüdischen] Lebens wie Fremdkörper“ (VE, 105) behandelt werden. Gleichzeitig deckt sie auf ihren Reisen wiederholt die zwar verdrängten, aber dennoch bestehenden Überlagerungen von jüdischem und nicht-jüdischem Leben auf, die sich vor allem räumlich, als eine Art ungewollte Nachbarschaft oder Ko-Habitation, äußern. Dies wird besonders deutlich während ihres Besuchs in der polnischen Stadt Kalizs: Ihre Begleiterin Pani Ania macht die Erzählerin darauf aufmerksam, „dass einige Straßen der Stadt mit Grabsteinen aus dem alten jüdischen Friedhof“ $(V E, 135)$ gepflastert sind. Ursprünglich wurden diese Grabsteine mit der Rückseite nach oben in die Straßen eingelassen, so dass sie nicht als Grabsteine zu erkennen waren; nach einer Erneuerung der Straßen zu einem späteren Zeitpunkt jedoch wurden sie unbeabsichtigterweise zum Teil mit der Vorderseite nach oben verlegt. Diese als Pflastersteine missbrauchten jüdischen Grabsteine fungieren nicht nur als Sinnbild für schockierende Pietätlosigkeit und die Missachtung des jüdischen Erbes im polnischen Kontext, sondern auch für die unausweichliche Wiederkehr des Verdrängten, das sich aus der Verflochtenheit jüdischen und nicht-jüdischen Lebens und Sterbens vor und nach dem Krieg ergibt.

Es stellt sich im Hinblick auf diese Beobachtungen der Erzählerin die Frage, wie eine produktivere Form des Erinnerns aussehen könnte, die die kompli- 
zierte Geschichte polnisch-jüdischer Nachbarschaft nicht - im tatsächlichen wie im übertragenen Sinne - mit Füßen tritt. Darauf geben sowohl die Erzählerin in Vielleicht Esther als auch die Autorin Petrowskaja unter dem Stichwort der „gemeinsame[n] Erinnerung“ (VE, 191) eine Antwort, verstanden als eine Erinnerung, die sich nicht im Konkurrenzkampf von Opfergruppen oder der Abwehr von Schuld und Verantwortung aufreibt und erschöpft, sondern stattdessen ein Bewusstsein dafür schafft, dass man, laut der Autorin Petrowskaja, aus der Geschichte und den Nachwirkungen des Zweiten Weltkrieges „nicht aussteigen kann“ (Timm 2013):

Wir können über andere Dinge nachdenken, wir gehen dahin, dahin - irgendwann stolpern wir, möchten wir nicht, oder nicht [sic], ob es konkret dieses Thema ist oder Spuren in der Stadt oder, dass du deine Kinder so oder so erziehst. Es hat unglaubliche Spuren hinterlassen im Verhalten, in der Kommunikation der Menschen. (Timm 2013)

Es geht also weniger um Schuldzuweisungen oder die Generierung von symbolischem Kapital durch die Vereinnahmung der Opferrolle, sondern um eine Offenheit und Aufnahmefähigkeit für die „Spuren“ und das Insistieren der Vergangenheit, die etwas geschaffen hat, was die Autorin als „unsere gemeinsame Antike“ bezeichnet. Wie Jessica Ortner argumentiert, ersetzt Petrowskajas Schreiben ein segregierendes Erinnern, das, um eine Formulierung Michael Rothbergs zu verwenden, leicht in ein ,zero-sum game of competition“ zwischen verschiedenen Opfergruppen ausarten kann (Rothberg 2009, 9) durch einen „gemeinsamen“ oder inklusiven Ansatz: „Throughout the entire novel, selection as opposed to inclusion is used as a metaphor for the segmentation of cultural memory, which Petrowskaja defines as a typical characteristic of post-socialist memory culture“ (Ortner 2017, 44). Lucia Perrone Capano spricht in diesem Zusammenhang auch von einer „verknüpfenden Erinnerungsarbeit“ (2018, 106). „Gemeinsam“ bedeutet in diesem Kontext auch, den Zweiten Weltkrieg und den Holocaust nicht als national gerahmte, sondern als transnationale und letzten Endes europäische Ereignisse wahrzunehmen und eine Art europäisches Gedächtnis zu schaffen, wie es der Text Vielleicht Esther versucht (zu europäischen und transnationalen Gedächtnisdiskursen im Text siehe Ortner 2017; Perrone Capano 2018). ${ }^{9}$

\footnotetext{
9 Es ist hierbei zu beachten, dass das Beharren auf „gemeinsamer Erinnerung“ und einem europäischen Gedächtnisraum in Petrowskajas Text und in Interviews wiederum ganz eigene Exklusionsmechanismen zu Tage fördert, die den Zusammenhang zwischen der (Erinnerung an die) Ermordung der Jüd*innen Europas und der (Nach-)Geschichte des Kolonialismus in Europa ausblenden. Zum Zusammenhang von Holocaust-Erinnerung und (Post-)Kolonialismus siehe vor allem Craps 2013; Rothberg 2009; Sanyal 2015; Silverman 2013.
} 
Ein solch europäisches Bewusstsein und Gedächtnis sind jedoch aus der Perspektive des Textes Vielleicht Esther (und auch ansonsten) ein ferner Traum; ein Umstand, dessen sich auch die Erzählerin schmerzlich bewusst ist: „,...] sogar im Gedenken setzt die Selektion sich fort“ $(V E, 191)$. Die „gemeinsame Erinnerung“ erscheint also realpolitisch nicht umsetzbar, dafür ist sie jedoch künstlerisch darstellbar: Es ist kein Zufall, dass Vielleicht Esther geprägt ist durch eine Metaphorik und Poetik des Klöppelns, Strickens, oder Webens (siehe hierzu Roca Lizarazu 2018), die unsichtbare Vernetzungen sichtbar macht und neue Verknüpfungen schafft und damit auf der Ebene der Form reflektiert, was Rothberg (2013, 2014a, 2014b) unter dem Schlagwort implication - was sich auf deutsch als „Verwicklung“ oder „Verstrickung“ übersetzen lässt - theoretisch zu denken versucht.

Zum einen finden sich im Text zahlreiche Motive der Vernetzung, etwa wenn die Erzählerin ihre Reisen als eine Suche nach den ,inneren Verbindungen [ihrer] Familie“ $(V E, 120)$ beschreibt, also als einen Versuch, Verknüpfungen offenzulegen aber mitunter auch erst herzustellen. Noch offensichtlicher tritt die Metaphorik des Gewebes in den zahlreichen Verweisen auf das Internet - im Englischen als World Wide Web bekannt - zu Tage. Das Internet wird von der Erzählerin als ein Medium präsentiert, das unterschiedliche Menschen, Orte und Geschichten im wahrsten Sinne des Wortes vernetzt und auf diese Weise Verkettungen aufzeigt oder überhaupt erst produziert, etwa wenn die Erzählerin in der OnlineDatenbank von Yad Vashem auf eine (mögliche) entfernte Verwandte, Mira Kimmelmann, stößt (VE, 118-128).

Solche Praktiken der Verwebung sind jedoch nicht nur für die Motivik und Bildsprache, sondern auch für die Poetik der Geschichtensammlung Vielleicht Esther zentral, die von Lucia Perrone Capano als „Buch der Verflechtungen“ $(2018,98)$ bezeichnet wird. Vielleicht Esther bleibt damit der Herkunft des Wortes „Text“ treu, das von lateinisch textum abstammt, was Gewebe oder Geflecht bedeutet. Diese Verflechtungen bestehen zum einen zwischen Petrowskajas Buch und diversen anderen Texten, die Vielleicht Esther in Form intertextueller Fäden durchziehen. Wichtig sind insbesondere wiederkehrende Bezüge auf die griechischen Mythen und die homerischen Epen: die Suche der Erzählerin ist nicht nur „eine Reise in das Labyrinth der Geschichte“ (Perrone Capano 2018, 100), in dem sie sich mit Hilfe des „Ariadnefaden[s]“ des Erzählens zu orientieren sucht $(V E, 62)$, ihre Reisen gemahnen auch an die Odyssee und ihre Großmutter väterlicherseits wird beschrieben als „Penelope“ (VE, 19); gleichzeitig identifiziert die Erzählerin sich auch mit Achilles und seiner verletzlichen Ferse (VE, 216-217). Verdichtet wird das Textgeflecht von Vielleicht Esther zum anderen durch die Mehrsprachigkeit des Buches, das neben dem Deutschen auch Versatzstücke aus dem Englischen, Jiddischen, Polnischen, Russischen und Ukrainischen beherbergt (siehe zum Thema Mehrsprachigkeit auch Bühler-Dietrich 
2019; Eckart 2015; Weiss-Sussex 2017). Das Schreiben in „der Kluft der Sprachen“ $(V E, 115)$ ermöglicht es der Erzählerin, einen im positiven Sinne heimatlosen, „perfekt verloren[en]“ $(V E, 115)$ Standpunkt einzunehmen, der auch die oben erwähnte, „gemeinsame Erinnerung“ begünstigt, denn Festlegungen und Grenzziehungen werden gelockert: „Wer hat wen erobert, wer gehört zu den Meinen, wer zu den anderen, welches Ufer ist meins?“ $(V E, 115) .{ }^{10}$ Damit eng verbunden ist ein assoziativer Zugang zu Sprache, der zahlreiche linguistische Verflechtungen erzeugt, etwa wenn die Erzählerin über den Fikus berichtet, der während der Flucht der Familie aus Kiew ihrem Vater auf dem Lastwagen weichen musste und zurückgelassen wurde und dem sie somit auch ihr eigenes Leben verdankt (VE, 216-220). Es bleibt unklar, ob sich die Geschichte mit dem Fikus wirklich so zugetragen hat, gleichzeitig ist er jedoch bestimmend für das Herkunftsnarrativ der Erzählerin, weshalb die Pflanze zwischen Fiktion, „Fixpunkt“ $(V E, 217)$ und Fixierung changiert. Die Verkettung dieser Wörter, die zuerst einmal über lautliche Ähnlichkeit funktioniert, verweist damit auf tieferliegende Zusammenhänge. Wie Godela Weiss-Sussex überzeugend argumentiert, ermöglicht der assoziative Sprachgebrauch im Text nicht nur neue Verbindungen und ein multiperspektivisches Narrativ (Weiss-Sussex 2017), er schärft auch das Gespür der Leser`innen für sprachliche Nach- und Missklänge, Sedimente und Verdrängtes, also das im Begriff implication auch mitschwingende Implizite, beispielsweise, wenn sich die Erzählerin nach ihrem Besuch in Auschwitz partout nicht „konzentrieren“ kann $(V E, 59)$ oder von ihren Schwierigkeiten „eine Einstellung zur Arbeit zu finden“ $(V E, 60)$ berichtet, da das deutsche Wort sich für sie nicht von der Toraufschrift von Auschwitz, „Arbeit macht frei“, trennen lässt. Wie Elin Nesje Vestli anmerkt, ist Petrowskajas Sprachgebrauch grundsätzlich vielschichtig und mehrdeutig, bei ihr „schreiben [...] die anderen Sprachen mit“ $(2016,155)$. Ebenso schreiben aber auch die anderen Texte, Erinnerungskulturen und Bewusstseinsebenen mit, so dass Literatur, Sprache und Gedächtnis als Palimpseste begreifbar werden. Damit übersetzt der Text die palimpsestischen Überlagerungen, die das Erinnern und die Topografien im Text (Berlin, Kalisz, Warschau, Babij Jar) prägen, in eine angemessene sprachliche Form. ${ }^{11}$

10 Annette Bühler-Dietrich merkt außerdem richtigerweise an, dass die Mehrsprachigkeit im Text einen „Ausweg aus der offiziellen russischen Sprache der Politik und der Geschichtsschreibung“ (2019, 235) bietet, die im Text mit festgezurrten Täter- und Opfernarrativen, Verdrängung und (staatlich verordnetem) Vergessen assoziiert ist.

11 Im Hinblick auf das Schaffen des südafrikanischen Künstlers William Kentridge merkt Michael Rothberg an, dass „palimpsest, morphing, and procession“ (2012, 9) als wesentliche Strategien für die künstlerische Darstellung und Verarbeitung von einer ,implicated“ Perspektive 
Somit tritt das Gewebe nicht zuletzt auch als zentrale poetologische Metapher hervor, denn Erzählen, verstanden als die Freilegung und Erzeugung von Verknüpfungen, wird im Text immer wieder mit „Handarbeit“, insbesondere mit Spinnerei gleichgesetzt: „Ich sollte spinnen, beherrschte aber keine Handarbeit“ (VE, 134). In diesem Zitat schwingen nicht nur erneut Referenzen auf diverse Gestalten aus den antiken Mythen und Epen mit, die allesamt mit Praktiken des Spinnens oder Webens verknüpft sind (Arachne, Ariadne, Athene, Clotho, Penelope), sondern auch Anklänge an die deutsche Formulierung „Garn spinnen“ oder „sich etwas zusammenspinnen“ für Lügen oder Fantasieren oder sogar „spinnen“ im Sinne von verrückt sein. Diese Konnotationen greifen das für den Text zentrale Verhältnis von Fakt und Fiktion, Wahrheit und Lüge auf. Indem die Erzählerin anmerkt, dass sie „keine Handarbeit“ beherrsche, verweist sie zudem darauf, dass sie abgeschnitten ist vom Familienerbe, das über Jahrhunderte darin bestand, taubstummen Kindern mittels Zeichensprache das Sprechen beizubringen. Insofern ergibt das von der Erzählerin gestaltete Textgewebe Vielleicht Esther denn auch keine ebenmäßigen Muster, sondern lediglich „kleine Fetzen“ (VE, 134) in Gestalt der fragmentarischen „Geschichten“ und ,verlorene[n] Fäden“ (VE, 134), die sich nicht zu einer kohärenten Familienerzählung zusammenfügen lassen.

Die formale Gestaltung des Textes ist demnach untrennbar mit den Themen Nachbarschaft und implication verbunden, indem eben genau jene Verknüpfungen, Verkettungen und Verstrickungen, die im offiziellen Gedächtnis häufig verdrängt werden, zum Stilprinzip erhoben werden. Dies zeigt sich darin, dass die Geschichte des Zweiten Weltkriegs und des Holocausts nicht als teleologisch angelegte Meistererzählung, sondern als Gewebe aus „kleinen Fetzen“ präsentiert wird, gekennzeichnet durch Fragmentarizität, zeitliche Sprünge, unerwartete Verknüpfungen und zahlreiche Abzweigungen und Seitenpfade.

Das zu Beginn dieses Abschnitts angeführte Zitat aus Vielleicht Esther verdeutlicht, wie Nachbarschaft und implication zum einen ein anderes, gemeinsames' Erinnern und zum anderen neue Erzählweisen notwendig werden lassen. Abschließend soll darauf eingegangen werden, dass eine „implicated“ Perspektive auch die Problematik der Zeugenschaft in einem neuen Licht erscheinen lässt. Da es sich bei implicated subjects (Rothberg 2014a) um Personen handelt, die entweder ihre Teilnahme am Geschehen zu verdrängen wünschen (beispielsweise im Falle der Mittäter`innen und Kompliz`innen) oder (angeblich) nicht bewusst an den Ereignissen teilgenommen haben (so z.B. Nachbar^innen, Passantinnen etc.), können (und wollen) diese Figuren nur bedingt Zeugnis ablegen. Dies wird

aufgefasst werden können; das Palimpsest und die assoziative Überblendung erscheinen also als mögliche Merkmale einer Poetik der implication. 
der Erzählerin besonders deutlich in dem Kapitel, das sich um Babij Yar und die titelgebende Urgroßmutter „Vielleicht Esther“ dreht. Da es keine Augenzeugenberichte zu den letzten Momenten im Leben ihrer Urgroßmutter gibt, ist die Erzählerin auf Vermutungen und das bereits erwähnte konjunktivische Erzählprogramm angewiesen: „Wer flüstert uns Geschichten ein, für die es keine Zeugen gibt?“ (VE, 221). Diese Problematik gewinnt an Schärfe im weiteren Kontext des Babij Jar-Massakers, da es kaum Überlebende gab und die Erinnerung an die Ereignisse von Seiten des sowjetischen und später ukrainischen Staates über Jahrzehnte systematisch verdrängt wurde. Ein offizielles Denkmal für die jüdischen Opfer des Massakers wurde erst im Jahr 1991 errichtet und bis heute wird die Tatsache, dass die Opfer von Babij Jar beinahe ausschließlich aufgrund ihrer jüdischen Herkunft ermordet wurden, im offiziellen Gedächtnisdiskurs weitgehend ausgeblendet (zur Geschichte Babij Jars als Erinnerungsort siehe Rapson 2014, 2015). Was bleibt angesichts dieser kollektiven Amnesie, ist ein Netzwerk ,unsichtbarer Zeugen“ (VE, 222), das jedoch weder für die Erzählerin noch für die Geschichtsschreibung zugänglich ist:

[...] Passanten, die Verkäuferinnen in der Bäckerei drei Stufen tiefer und die Nachbarn hinter den Vorhängen dieser dicht bewohnten Straße, eine nirgendwo erwähnte, gesichtslose Masse für die großen Flüchtlingszüge. Sie sind die letzten Erzähler. Wohin sind die alle umgezogen? (VE, 222)

Was sich hier auftut, ist eine ungeschriebene und vermutlich auch nie zu schreibende Geschichte des Zweiten Weltkriegs und des Holocausts, die ein ästhetisches, epistemologisches und, im Hinblick auf die kollektiv verordnete Amnesie im sowjetischen Kontext, letztlich auch ethisches und politisches Problem darstellt. Der Text Vielleicht Esther interveniert in diese Problematik zum einen durch den selbstreflexiven Einsatz von Fiktion an jenen Stellen, an denen das Faktische notwendigerweise an seine Grenzen stößt, und zum anderen durch eine alternative Erinnerungspolitik, die wesentliche Elemente des Nachbarschaftlichen - nämlich Kontiguität, Kontingenz und (Über-)Kreuzung - als Ausgangspunkt für neue, inklusive und nicht essentialisierende Formen der Vergemeinschaftung nimmt.

\section{Kontiguität, Kontingenz und (Über-)Kreuzung}

Der vorliegende Beitrag hat sich vornehmlich dem Zusammenhang von Nachbarschaft und implication gewidmet, d.h. dem Problem der Verstrickung, das Opfer- und Täterrollen nachhaltig destabilisiert. In diesem Kontext sind Nachbarn vor allem als negative, oder doch zumindest ambivalente Figuren aufgetre- 
ten, weshalb es abschließend um mögliche ,positive“ Aspekte des Themas gehen soll. Wie bereits im Zusammenhang mit dem Kapitel zur Urgroßmutter „Vielleicht Esther“ angemerkt, gibt es im Text durchaus nachbarschaftliche Figuren, die helfen und positiv besetzt sind, so zum Beispiel, wenn die Erzählerin durch Zufall eine alte Nachbarin ihrer Mutter, Dina, wiederfindet, die das letzte, lebendige Verbindungsglied zur Vorkriegszeit darstellt $(V E, 86)$.

Wesentlich interessanter sind jedoch jene Aspekte des Textes, die Nachbarschaft als ein alternatives Ordnungs- und Relationsprinzip einführen, das auf Kontiguität, Kontingenz und dem für den Text wichtigen Motiv der (Über-)Kreuzungen beruht. Dieses Prinzip wird beispielsweise deutlich im Unterkapitel „Die Liste“ (VE, 25-29): während ihrer genealogischen Suche stößt die Erzählerin auf diverse Namenslisten, die möglicherweise die Namen ihrer Verwandten enthalten. Diese Listen sind im Zusammenhang des Familienromans, den Vielleicht Esther auch repräsentiert, interessant, da sie eine topografische und synchrone Dimension in das ansonsten diachrone, genealogisch strukturierte Such- und Erzählprogramm des Textes einführen. Im Gegensatz zum „Familienbaum“ (VE, 17-20), der das erste Kapitel von Petrowskajas Geschichtensammlung eröffnet, ist die Namensliste nicht an zeitlicher Generationenabfolge und damit der Nähe oder Distanz zu einem irgendwie definierten Ursprung orientiert. Zusätzlich ist sie nicht auf einige wenige, dem engeren Familienkreis zugehörige Individuen konzentriert, sondern besteht aus einer Ansammlung von Namen ohne Gesicht und Geschichte - man kann die Namen auf der Liste zählen, jedoch nicht erzählen, wie Sigrid Weigel angemerkt hat (2006). Alle auf der Liste befindlichen Individuen nehmen zudem dieselbe zeitliche Ebene ein, denn bei einer Liste geht es um Klassifikation und Synchronie, nicht um zeitliche Abfolge und Diachronie. Die Gruppierung der Namen auf der Liste basiert auf einem gemeinsamen und gleichzeitig doch kontingenten Merkmal - wie beispielsweise die jüdischen Opfer des Holocaust, die Opfer des Zweiten Weltkriegs etc. -, aber aus dieser Anordnung lässt sich keine Kausalität und kein Narrativ ableiten, denn eine Erzählung bedarf zeitlicher Abfolge. Das räumliche Konzept der Nachbarschaft, verstanden im Sinne von Kontiguität, ersetzt damit das zeitlich gebundene Konzept der Abstammung: „[...] in den Listen stehen sie alle untereinander, nebeneinander wie Nachbarn, durcheinandergemischt, und die Meinigen sind nicht zu unterscheiden von Hunderten anderer, die genauso heißen, [...]“ (VE, 27). Das räumlich kodierte ,[U]ntereinander, [N]ebeneinander“ $(V E, 27)$ der Nachbarschaft steht im Gegensatz zum zeitlichen Prinzip des Nacheinanders in der Geschlechterabfolge und stellt somit für das genealogische Programm der Erzählerin ein Hindernis dar, da es eine fundamental andere Logik und ein anderes Ordnungssystem repräsentiert: „Je mehr Gleichnamige es gab, desto geringer war die Chance, meine Verwandten unter ihnen zu finden [...]“ $(V E, 27)$. Die Erzählerin reagiert auf diese Herausforderung, indem sie sich auf ein 
anderes, nicht rein biologisch definiertes Verständnis von Familie einlässt: „[...] und je geringer diese Chance war, desto klarer wurde mir, dass ich alle Aufgelisteten zu den Meinigen zu zählen hatte“ (VE, 27).

Die Namensliste und die mit ihr verbundenen Themen der Nachbarschaft und des räumlichen Nebeneinanders muss daher auch im Kontext der weiter oben erwähnten Erinnerungsproblematik gesehen werden. Vielleicht Esther lotet nicht nur die Grenzen des institutionalisierten, sondern auch des familiären Gedächtnisses aus (siehe hierzu auch Ortner 2017; Roca Lizarazu 2018), das genauso wie der offizielle historiografische oder politische Diskurs Exklusionen erzeugt. Der Text präsentiert Nachbarschaft als ein alternatives Ordnungs- und Verbindungsprinzip, das zeitliche Abfolgen und Ursprungsdenken, und also „Genea-Logik“ (Weigel 2006), durch räumliche Lagerung und mithin einen topografischen Zugang ersetzt. Durch diese Operation wird das ermöglicht, was Marianne Hirsch als „affiliative“ Formen der postmemory beschreibt (1997, 2008, 2012): gemeint sind damit all jene Formen des Nachgedächtnisses, die nicht auf der ,intergenerational vertical identification of child and parent occurring within the family“ (Hirsch 2012, 36) beruhen, d.h. Formen der Identifikation, die auf Adoption und Wahlverwandtschaften fußen. Eröffnet werden dadurch Möglichkeiten der Erinnerungsvernetzung, die auch jener Opfer gedenken, die nicht zum unmittelbaren Kreis der biologisch (oder ethnisch, national etc.) definierten „Meinigen“ $(V E, 27)$ gehören. Der Zusamenhang von Nachbarschaft, ,affiliation“ und (post-)familiärer Erinnerungsgemeinschaft fügt damit dem weiter oben erwähnten Programm der „gemeinsame[n] Erinnerung“ eine zusätzliche Facette bei. Gemeint ist damit nicht nur eine „multidirectional“ Erinnerung im Sinne Michael Rothbergs (2009; Verweise auf Rothbergs Konzept der multidirectional memory finden sich auch bei Perrone Capano 2018), die die Verstrickungen zwischen den unterschiedlichen Gewaltereignissen des zwanzigsten Jahrhunderts anerkennt, sondern auch ein bis zu einem gewissen Grad postfamiliäres Erinnern, das mit der Exklusivität des Familiengedächtnisses bricht. Diese Erinnerungspolitik des Textes Vielleicht Esther gewinnt an Sprengkraft im Kontext der oben erwähnten, staatlich verordneten Erinnerungs- und Vergessenspolitik im (post-)sowjetischen Raum, die sich systematisch weigert, bestimmte Opfergruppen anzuerkennen. Das zufallsgesteuerte Prinzip der Nachbarschaft bewirkt damit eine Problematisierung und, zumindest teilweise, ${ }^{12}$ Verabschiedung essentialisierender (Erinnerungs-)

12 Auch wenn Vielleicht Esther alternative, d.h. nicht biologisch oder ethnisch determinierte Formen der Erinnerungsgemeinschaft einführt, bleibt der Text als Ganzes doch am genealogischen Grundprojekt orientiert; außerdem orientieren sich die Grenzen der „affiliativen“ Inklusionen letzten Endes an Opfer- und Täterzuschreibungen, siehe hierzu auch Roca Lizarazu 2018. 
Gemeinschaften, die auf familiärer, ethnischer oder nationaler Zugehörigkeit beruhen und notwendigerweise Ausschlüsse erzeugen. Nachbarschaftsverhältnisse hingegen sind fundamental anti-essentialistisch, denn verbunden sind Nachbarn nicht durch eine irgendwie geartete Wesenshaftigkeit, sondern durch das zufällige und oft auch flüchtige Nebeneinander im Raum.

Das Motiv der zufälligen Überkreuzung findet sich an diversen weiteren Stellen im Text, so zum Beispiel im Unterkapitel „Nachbarn“ (VE, 40-44). In diesem Kapitel beschreibt die Erzählerin den Kiewer Wohnblock, in dem sie aufwuchs. Obwohl die Bekanntschaften nach Aussagen der Erzählerin „nur flüchtig“ (VE, 42) sind, versammeln sie doch ein „multidirectional“ (Rothberg, 2009) Panorama der Gewalterfahrungen des zwanzigsten Jahrhunderts, das vom Spanischen Bürgerkrieg über den Zweiten Weltkrieg bis hin zum Holocaust reicht. Auch in diesem Falle wird also über Nachbarschaften eine nicht-essentialistische Lebens-, Leidens- und Erinnerungsgemeinschaft hergestellt, derer sich die Mitglieder mitunter nicht einmal bewusst sind. Solcherlei unbewusste Überkreuzungen und „Zufälle in Raum und Zeit“ (VE, 70) spielen für Vielleicht Esther eine zentrale Rolle, etwa wenn die Erzählerin über die - tatsächliche und metaphorische - Nachbarschaft zwischen ihrem Urgroßvater Ozjel und dem berühmten polnischen Pädagogen Janus Korczak schreibt:

[...] wieder waren sie Nachbarn in Raum und Zeit, ohne es zu wissen, er [Korczak] schrieb in Kiew, weil ihn der Erste Weltkrieg in meine Stadt gebracht hatte, dann kehrte er nach Warschau zurück. Wären mein Urgroßvater Ozjel und meine Großmutter Rosa damals zurückgegangen, wären sie und ihre Waisenhäuser, ihre Waisen und ihre eigenen Kinder, Nachbarn von Janus Korczak geworden, mit allen folgenden Adressen. (VE, 71)

Erneut zeigt sich hier die Ambivalenz des Nachbarschaftsbegriffes, denn wären der Urgroßvater und die Großmutter der Erzählerin die Nachbarn Korczaks geblieben, wären sie mit ihm erst im Warschauer Ghetto und dann im Vernichtungslager Treblinka gelandet. Gleichzeitig wird die räumliche Nähe zwischen der Teilfamilie der Erzählerin und Korczak zum Anlass genommen, um auf bestehende Gemeinsamkeiten zu verweisen (Judentum, heilpädagogischer Hintergrund) oder neue zu erzeugen, denn die Anekdote zur Nachbarschaft wird auch zum Anlass des Eingedenkens - Korczak und seine Waisen werden in die Erinnerungsgemeinschaft der Erzählerin (und möglicherweise auch der Leser`innen) aufgenommen. Darüber hinaus ist das Beispiel Korczaks nicht zufällig gewählt, da der polnische Pädagoge sich bekanntermaßen bewusst dazu entschied, mit den von ihm betreuten Waisenkindern in den Deportationszug nach Treblinka zu steigen, obwohl er die Möglichkeit gehabt hätte, sich zu retten. Das Beispiel Korczaks greift damit die den Text prägende Thematik der Adoption, der Wahlverwandschaft und der alternativen Familiensysteme auf. 
Wie die Erzählerin anmerkt, sind sich die Beteiligten dieser Nachbarschaften und (Wahl-)Verwandtschaften mitunter nicht bewusst - sie werden sichtbar gemacht (oder auch erst erzeugt) als Produkte der assoziativen, vernetzenden Poetik des Textes Vielleicht Esther. Literatur tritt damit als wesentliches Medium hervor, um die hier beschriebene, anti-essentialistische Logik und Politik des Nachbarschaftlichen zu veranschaulichen und umzusetzen.

\section{Schluss}

Der vorliegende Beitrag hat versucht, sich über einen Nebenpfad, nämlich über Figuren und Figurationen des Nachbarschaftlichen, zentralen Themen im Text von Petrowskaja zu nähern: Opfertum und Täterschaft, Komplizenschaft und implication, Gedächtnis und Poetologie. Es hat sich gezeigt, dass Nachbarschaftsverhältnisse in Petrowskajas Text untrennbar mit dem verbunden sind, was Michael Rothberg als implication beschreibt, also Formen der Partizipation an Gewaltereignissen, die nicht im Opfer-Täter-Schema aufgehen, und damit Fragen der Komplizität und Kollaboration, aber auch der Nutznießerschaft und der Erboder Hinterlassenschaft aufwerfen.

Nachbarschaftsverhältnisse dienen in Vielleicht Esther dazu, im Sinne einer erinnerungspolitischen Intervention sowohl Opfer- als auch Täterrollen zu destabilisieren. Indem darauf verwiesen wird, dass Jüd*innen und Nicht-Jüd*innen vor und während des Zweiten Weltkriegs Nachbarn waren, wird die Distanz aufgehoben, die notwendig wäre, um diese beiden Gruppen voneinander getrennt $\mathrm{zu}$ halten und als die jeweils ,anderen' zu etikettieren - diese Intimisierung des jüdisch-nicht-jüdischen Verhältnisses hat jedoch im Hinblick auf Jüdinnen und Nicht-Jüd^innen jeweils unterschiedliche Effekte: Im Falle der ukrainischen und polnischen Bevölkerung wird das Nachbarschaftsverhältnis zwischen Jüd*innen und Nicht-Jüd*innen von der Erzählerin zum Anlass genommen, um das in diesen Gesellschaften nach wie vor tabuisierte Thema der Kollaboration, Mittäterschaft und Nutznießerschaft zu durchdenken. Damit werden zum einen nationale Opfernarrative in Frage gestellt, die jegliche Mitverantwortung für die Verbrechen des Holocausts abwehren helfen und die lange Vorgeschichte antisemitischer Ausschreitungen in beiden Ländern ausblenden; zum anderen wird aber auch aufgezeigt, dass die starre Opposition von ,Opfern' und ,Täter*innen wenig hilfreich ist, um Prozesse zu verstehen, die sich oft in den Grauzonen zwischen diesen beiden Zuschreibungen abspiel(t)en.

Im Hinblick auf die jüdischen Opfer des Holocaust eröffnet das Thema Nachbarschaft „affiliative“ Zugänge, um Marianne Hirsch zu zitieren (1997, 2008, 2012), die eine Erweiterung der vornehmlich familiären und ethnisch kodierten 
Erinnerungsgemeinschaften im Text erlauben. Das Prinzip der Nachbarschaft, das auf räumlicher Nähe und Synchronie beruht, komplementiert das die Erzählung dominierende, auf Abstammung und Diachronie fußende, genealogische Prinzip auf produktive Weise. Dies ist auch wichtig im Kontext der für den Text zentralen (post-)sowjetischen Gedächtnispolitiken, die die jüdischen Opfer des Holocaust lange Zeit nicht anerkannt haben und, laut der Erzählerin, nach wie vor stiefmütterlich, als „die Toten der ewigen anderen“ (VE, 190) behandeln. Statt also eine ähnlich exklusive, auf biologischer oder ethnischer Gemeinsamkeit beruhende Erinnerungspolitik zu betreiben, vertritt Vielleicht Esther einen nicht-essentialisierenden, nachbarschaftlichen Ansatz, der nicht zwischen ,den Meinigen' und den ,anderen' unterscheidet und mithin diskriminiert. Das Nachbarschaftliche steht damit im Zentrum einer Politik, die, ,als Gegenbewegung zu einer parzellierten Erinnerungskultur“ (Bühler-Dietrich 2019, 245) von der Autorin Petrowskaja unter dem Schlagwort der „gemeinsame[n] Erinnerung“ gefasst wird. „Gemeinsam“ schließt dabei „implicated“ oder „multidirectional“ Ansätze im Sinne Michael Rothbergs (2009, 2013, 2014a, 2014b) ein, die auf der gegenseitigen Bedingtheit von Vergangenheit, Gegenwart und Zukunft sowie der Verflochtenheit zwischen den europäischen und außereuropäischen Gewalterfahrungen des zwanzigsten Jahrhunderts beharren, ebenso wie ein Erinnern, das, im Sinne der „affiliation“ (Hirsch 1997, 2008, 2012), bewusst mit der notwendigen Exklusivität ethnischer, familiärer oder nationaler Gedächtnisrahmen bricht.

Es wird also deutlich, dass, in Entsprechung zum assoziativen Schreibprinzip des Textes, das Thema Nachbarschaft untrennbar mit den zentralen Themen des Textes verknüpft ist: Neben Fragen von Opfertum, Täterschaft und Erinnerung betrifft dies auch die Ebene der Poetik. Durch eine Metaphorik und Poetik der Vernetzung und einen assoziativen Sprachgebrauch setzt Vielleicht Esther Michael Rothbergs Gedanken zu implication künstlerisch um und verdeutlicht damit, dass die „gemeinsame Erinnerung“ auch vor allem eine literarische Erinnerung ist, die dort ansetzt, wo offizielle Gedächtnisdiskurse in binären Mustern und damit im Modus der Exklusivität und der „Selektion“ (VE, 191) erstarren. Dies legt den Schluss nahe, dass das Medium der Literatur und der Kunst im Allgemeinen womöglich besonders geeignet ist, um nicht nur alternative, sondern auch zukunftsträchtigere Erinnerungsformen und -gemeinschaften zu entwerfen. 


\section{Literaturverzeichnis}

Bühler-Dietrich, Annette. „,Hast du nicht alle zu Hause': Sprache und Gleichgewicht in Katja Petrowskajas Vielleicht Esther". Affektivität und Mehrsprachigkeit: Dynamiken in der deutschsprachigen (Gegenwarts-)Literatur. Hg. Marion Acker, Anne Fleig und Matthias Lüthjohann. Tübingen: Narr, 2019. 225-246.

Costagli, Simone, und Matteo Galli. Hg. Familienromane: Literarische Genealogien und internationaler Kontext. München: Wilhelm Fink, 2010.

Craps, Stef. Postcolonial Witnessing: Trauma out of Bounds. New York: Palgrave Macmillan, 2013. Dudek, Bartosz. „Holocaust-Gesetz: In Kraft, aber eingefroren?“. dw.com, 28. Februar 2018, https://www.dw.com/de/holocaust-gesetz-in-kraft-aber-eingefroren/a-42776674 (8. Oktober 2018).

Eckart, Gabriele. „The Functions of Multilingual Language Use in Katja Petrowskaja's Vielleicht Esther“. Glossen 40 (2015), http://blogs.dickinson.edu/glossen/archive/most-recent-issüglossen-402015/gabriele-eckart-glossen40-2015/ (12. Oktober 2018).

Eichenberg, Ariane. Familie - Ich - Nation: Narrative Analysen zeitgenössischer Generationenromane. Göttingen: Vandenhoeck \& Ruprecht, 2009.

Eigler, Friederike. Gedächtnis und Geschichte in Generationenromanen seit der Wende. Berlin: Erich Schmidt, 2005.

Eßlinger Eva, Tobias Schlechtriemen, Doris Schweitzer und Alexander Zons. Hg. Die Figur des Dritten: Ein kulturwissenschaftliches Paradigma. Frankfurt a. M.: Suhrkamp, 2010.

Fuchs, Anne. Phantoms of War in Contemporary German Literature, Films and Discourse: The Politics of Memory. New York: Palgrave Macmillan, 2008.

Gross, Jan T. Neighbours: The Destruction of the Jewish Community in Jedwabne, Poland. Princeton, NJ und Oxford: Princeton University Press, 2001.

Harper, Jo. „Bezeichnung ,polnische Konzentrationslager“ bald strafbar“. dw.com, 18. August 2016, https://www.dw.com/de/bezeichnung-polnische-konzentrationslager-baldstrafbar/a-19480119 (3. Januar 2019).

Heimann, Holger. „Deutsche Sprache kam einer Befreiung gleich“. welt.de, 8 Juli 2013, https:// www.welt.de/kultur/literarischewelt/article117810166/Die-deutsche-Sprache-kam-einerBefreiung-gleich.html (12. Oktober 2018).

Herrmann, Meike. Vergangenwart: Erzählen vom Nationalsozialismus in der deutschen Literatur seit den neunziger Jahren. Würzburg: Königshausen \& Neumann, 2010.

Hirsch, Marianne. Family Frames: Photography, Narrative, and Postmemory. Cambridge, Mass. und London: Harvard University Press, 1997.

Hirsch, Marianne. „The Generation of Postmemory“. Poetics Today 29.1 (2008): 103-128.

Hirsch, Marianne. The Generation of Postmemory: Writing and Visual Culture after the Holocaust. New York: Columbia University Press, 2012.

Horstkotte, Silke. Nachbilder: Fotografie und Gedächtnis in der deutschen Gegenwartsliteratur. Köln: Böhlau, 2009.

Koschorke, Albrecht. „Institutionentheorie“. Die Figur des Dritten: Ein kulturwissenschaftliches Paradigma. Hg. Eva Eßlinger, Tobias Schlechtriemen, Doris Schweitzer und Alexander Zons. Frankfurt a. M.: Suhrkamp, 2010. 49-64.

Lukács, Georg. „Die Theorie des Romans: Ein geschichtsphilosophischer Versuch über die Formen der großen Epik“. Zeitschrift für Ästhetik und allgemeine Kunstwissenschaft 11.3 (1916): 225-271. 
Ortner, Jessica. „The Reconfiguration of the European Archive in Contemporary German-Jewish Migrant-Literature“. Nordisk Judaistik/Scandinavian Jewish Studies 28.1 (2017): 38-54. Osborne, Dora. „Encountering the Archive in Katja Petrowskaja's Vielleicht Esther“. Seminar 52.3 (2016): 255-272.

Perrone Capano, Lucia. „Migrierende Geschichten: Transnationale Erinnerungsräume in Katja Petrowskajas Vielleicht Esther“. Interkulturelle Blicke auf Migrationsbewegungen in alten und neuen Texten. Hg. Raluca Radulescu, Lucia Perrone Capano, Nicoletta Gagliardi und Beatrice Wilke. Berlin: Frank \& Timme, 2018. 95-109.

Petrowskaja, Katja. Vielleicht Esther. Geschichten. Frankfurt a. M.: Suhrkamp, 2014.

Polonsky, Antony, und Joanna B. Michlic. Hg. The Neighbors Respond: The Controversy over the Jedwabne Massacre. Princeton, NJ und Oxford: Princeton University Press, 2004.

Rapson, Jessica. „Babi Yar: Transcultural Memories of Atrocity from Kiev to Denver“. The Transcultural Turn: Interrogating Memory between and beyond Borders. Hg. Lucy Bond und Jessica Rapson. Berlin und Boston: De Gruyter, 2014. 139-161.

Rapson, Jessica. Topographies of Suffering: Buchenwald, Babi Yar, Lidice. New York und Oxford: Berghahn Books, 2015.

Reidy, Julian. Rekonstruktion und Entheroisierung: Paradigmen des, Generationenromans ' in der deutschsprachigen Gegenwartsliteratur. Bielefeld: Aisthesis, 2013.

Roca Lizarazu, Maria. „The Family Tree, the Web, and the Palimpsest: Figures of Postmemory in Katja Petrowskaja's Vielleicht Esther“. Modern Language Review 113.1 (2018): 169-189.

Rothberg, Michael. Multidirectional Memory: Remembering the Holocaust in the Age of Decolonization. Stanford: Stanford University Press, 2009.

Rothberg, Michael. „Progress, Progression, Procession: William Kentridge and the Narratology of Transitional Justice“. Narrative 20.1 (2012): 1-24.

Rothberg, Michael. „Multidirectional Memory and the Implicated Subject: On Sebald and Kentridge“. Performing Memory in Art and Popular Culture. Hg. Liedeke Plate. London und New York: Routledge, 2013. 39-58.

Rothberg, Michael. „Preface: Beyond Tancred and Clorinda: Trauma Studies for Implicated Subjects". The Future of Trauma Theory: Contemporary Literary and Cultural Criticism. Hg. Gert Buelens, Sam Durrant und Robert Eagleston. London und New York: Routledge, 2014a. XI-XVIII.

Rothberg, Michael. „Trauma Theory, Implicated Subjects, and the Question of Israel/Palestine“, Profession, 2. Mai 2014b, https://profession.commons.mla.org/2014/05/02/traumatheory-implicated-subjects-and-the-qüstion-of-isrälpalestine/ (12. Oktober 2018).

Sanyal, Debarati. Memory and Complicity: Migrations of Holocaust Remembrance. New York: Fordham University Press, 2015.

Sierdaka, Monika. „Polen entschärft kontroverses Holocaust-Gesetz“. dw.com, 27. Juni 2018, https://www.dw.com/de/polen-entsch\%C3\%A4rft-kontroverses-holocaustgesetz/a-44434647 (8. Oktober 2018).

Silverman, Max. „Memory Traces: Patrick Chamoiseau and Rodolphe Hammadi’s Guyane: Tracemémoire du bagne“. Yale French Studies 118/119 (2010): 225-238.

Silverman, Max. Palimpsestic Memory: The Holocaust and Colonialism in French and Francophone Fiction and Film. New York und Oxford: Berghahn, 2013.

Timm, Ulrike. „Es gibt keine Grenze zwischen Literaturen“. Deutschlandfunkkultur.de, 8. Juli 2013, https://www.deutschlandfunkkultur.de/es-gibt-keine-grenze-zwischenliteraturen.954.de.html?dram:article_id=252300 (12. Oktober 2018). 
Tzschentke, Hannah. „Motive der Verschränkung von Gegenwart und Vergangenheit in Katja Petrowskajas Vielleicht Esther“. Erschriebene Erinnerung: Die Mehrdimensionalität literarischer Inszenierung. Hg. Sanna Schulte. Köln: Böhlau, 2015. 270-286.

Vestli, Elin Nesje. „,Mein fremdes Deutsch“: Grenzüberschreitungen in der deutschsprachigen Gegenwartsliteratur - Katja Petrowskaja’s Vielleicht Esther". Language and Nation: Crossroads and Connections. Hg. Guri Ellen Barstad, Arnstein Hjelde und Sigfried Kvam. Münster und New York: Waxmann, 2016. 143-160.

Weigel, Sigrid. Genea-Logik: Generation, Tradition und Evolution zwischen Kultur- und Naturwissenschaften. München: Wilhelm Fink, 2006.

Weiss-Sussex, Godela. ,,Jeder Stern schien mir ein geheimer Verwandter zu sein': Heimat, Language, and Identity in Katja Petrowskaja, Vielleicht Esther (2014)“. Vortrag bei der 41. Annual Conference of the German Studies Association (US), Atlanta. 6. Oktober 2017.

Žižek, Slavoj, Eric L. Santner und Kenneth Reinhard. The Neighbour: Three Enquiries in Political Theology. Chicago und London: University of Chicago Press, 2005. 